\title{
On Discriminating between Gamma and Log-logistic Distributions in Case of Progressive Type II Censoring
}

\author{
Elsayed Ahmed Elsherpieny \\ Department of Mathematical Statistics, Institute of Statistical Studies and Research \\ Cairo University, Giza, Egypt \\ ahmedc55@yahoo.com \\ Hiba Zeyada Muhammed \\ Department of Mathematical Statistics, Institute of Statistical Studies and Research \\ Cairo University, Giza, Egypt \\ hiba_stat@yahoo.com \\ Noha Usama Mohamed Mohamed Radwan \\ Institute of Statistical Studies and Research, Cairo University, Giza, Egypt \\ nohausama@gmail.com
}

\begin{abstract}
Gamma and log-logistic distributions are two popular distributions for analyzing lifetime data. In this paper, the problem of discriminating between these two distribution functions is considered in case of progressive type II censoring. The ratio of the maximized likelihood test (RML) is used to discriminate between them. Some simulation experiments were performed to see how the probability of correct selection (PCS) under each model work for small sample sizes. Real data life is analyzed to see how the proposed method works in practice. As a special case of progressive type II censoring, the problem of discriminating between gamma and log-logistic in case of complete samples is considered. The RML and the ratio of Minimized Kullback-Leibler Divergence (RMKLD) tests are used to discriminate between them. The asymptotic results are used to estimate the PCS which is used to calculate the minimum sample size required for discriminating between two distributions. Two real life data are analyzed.
\end{abstract}

Keywords: Gamma distribution; Log-logistic distribution; Progressive type II censoring; Likelihood ratio; the ratio of minimized Kullback-Leibler divergence.

\section{Introduction}

Choosing the correct or best-fitting distribution for a given data set is an important issue. Most of the times distribution functions may provide a similar data fit but still it is desirable to select the correct or more nearly correct model. The effect of choosing the wrong model has been attempted by many researchers as Cox (1961), Wiens (1999) and Pascual (2005).

Special attention has been given to discriminate some specific distribution functions, due to the increase of their applications. Cox (1962) suggested tests of separate families of hypotheses and applied his test to discriminate between lognormal and exponential distribution. Atkinson (1970) combined Cox's two hypotheses in a general model and applied his test to discriminate between lognormal and exponential distribution. The ratio of the maximized likelihood (RML) procedure has been applied in discriminating between distributions by many authors as Dumonceaux et al. (1973), Dumonceaux and Antle (1973), Pereira (1977), Bain and Engelhardt (1980), Kappenman (1982), Firth 
(1988),. Pandey et al. (1991), Fearn and Nebenzahl (1991), Gupta et al. (2001). Gupta and Kundu (2003), Gupta and Kundu (2004), Kundu and Manglick (2005), Kundu and Raqab (2007), Bedar (2009), Dey and Kundu (2010), Ashkar and Aucion (2012), Raqab (2013), Elsherpieny et al. (2013), Rao and Kantam (2014).

Bromideh (2012), Bromideh and Valizadeh (2013) found that the Kullback-Leibler divergence (KLD) method works better than RML, because it generates higher probability of correct selection (PCS) in particular for small sample size. In other words, the error type I for RMKLD is remarkably less, compared to the RML one.

Some procedures for selecting between distributions for the cases of not only complete but also censored data in the cases of Type-I and Type-II censoring schemes have been paid attention by some authors, Siswadi and Quesenberry (1982), Kim et al. (2000), Cain (2002), Block and Leemis (2008), Kim and Yum (2008), Dey and Kundu (2009) and Dey and Kundu (2012). However, the conventional Type-I and Type-II censoring schemes do not have the flexibility of allowing removal of units at points other than the terminal point of the experiment. Because of this lack of flexibility, a more general censoring scheme called progressive Type-II right censoring has been introduced. Progressive censoring schemes are very useful in life-test experiments and in clinical studies. Some of the earlier work on progressive censoring and estimating the unknown parameters for different distribution functions were conducted by Cohen (1965), Mann (1971), Thomas and Wilson (1972), Viveros and Balakrishnan (1994), Balakrishnan and Sandhu (1995), Balasooriya and Balakrishnan (2000), Balakrishnan and Aggarwala (2000), Balakrishnan and Kannan (2001), Balakrishnan et al. (2003, 2004), Mousa and Jaheen (2002) and Balakrishnan (2007).

This paper consists of four Sections including this introduction, In Section 2 discriminating between Gamma and log-logistic distributions is considered in case of progressive type II censoring. In Section 3 the problem of discriminating between Gamma and log-logistic distributions is considered in case of complete sample. Finally paper conclusion in Section 4.

\section{Progressive Type II Censoring}

Under this scheme, $\mathrm{n}$ units are placed on a test at time zero, with $\mathrm{m}$ failures to be observed. When the first failure is observed, $r_{1}$ of the surviving units are randomly selected and removed. At the second observed failure, $r_{2}$ of the surviving units are randomly selected and removed. This experiment stops at the time when the $\mathrm{m}$-th failure is observed and the remaining $r_{m}=n-r_{1}-r_{2}-\ldots-r_{m-1}-m$ surviving units are all removed.

In this scheme, $r_{1}, r_{2}, \ldots, r_{m}$ are pre-determined. Thus, here the censoring times (Ti's) are random, but the numbers of items to fail before each censoring time are fixed. The resulting $\mathrm{m}$ ordered values which are obtained are referred to as progressively type II right censored order statistics. [Balakrishnan and Aggarwala (2000)]. 


\subsection{Maximum Likelihood Estimation for Gamma Distribution}

The probability density function of the gamma distribution, denoted by $\operatorname{GA}(\lambda, \alpha)$, with scale parameter $\lambda>0$ and shape parameter $\alpha>0$ is given by

$$
f_{G A}(x ; \lambda, \alpha)=\frac{1}{\lambda^{\alpha} \Gamma(\alpha)}(x)^{\alpha-1} e^{-\left(\frac{x}{\lambda}\right)} \quad \lambda, \alpha>0 \quad x>0 .
$$

Let $\mathrm{X}_{1: \mathrm{m}: \mathrm{n}}, \ldots, \mathrm{X}_{\mathrm{m}: \mathrm{m}: \mathrm{n}}$ be progressively type II censored sample from a two parameter Gamma distribution, with censoring scheme $r=\left(r_{1}, \ldots, r_{m}\right)$. The likelihood function is given by

Where

$$
L_{G A}(\alpha, \lambda)=k \prod_{i=1}^{m} f\left(x_{i: m: n}\right)\left[1-F\left(x_{i: m: n}\right)\right] r_{i}
$$

$$
K=n\left(n-1-r_{1}\right)\left(n-2-r_{1}-r_{2}\right) \ldots\left(n-m+1-r_{1}-\ldots-r_{m-1}\right) .
$$

For simplicity of notation, we will use $x_{i}$ instead of $x_{i: m: n}$.

Then likelihood function may then be written as,

$$
\begin{aligned}
L_{G A}(\alpha, \lambda) & =k \prod_{i=1}^{m} \frac{1}{\lambda^{\alpha} \Gamma(\alpha)} x_{i}^{\alpha-1} e^{-\frac{x_{i}}{\lambda}}\left[1-F_{G A}\left(x_{i}, \lambda, \alpha\right)\right]_{i}^{r} \\
& \propto \frac{1}{\lambda^{m \alpha}(\Gamma(\alpha))^{m}} \prod_{i=1}^{m} x_{i}^{\alpha-1} \prod_{i=1}^{m} e^{-\frac{x_{i}}{\lambda}} \prod_{i=1}^{m}\left[1-\frac{\gamma\left(\alpha, \frac{x_{i}}{\lambda}\right)}{\Gamma(\alpha)}\right]^{r} i
\end{aligned}
$$

Where

$$
\gamma\left(\alpha, \frac{x_{i}}{\lambda}\right)=\int_{0}^{t}\left(\frac{x_{i}}{\lambda}\right)^{\alpha-1} e^{-\left(\frac{x_{i}}{\lambda}\right)} d t
$$

Then log likelihood function may then be written as,

$$
\begin{aligned}
\ln L_{G A}(\alpha, \lambda)=- & m \ln (\Gamma(\alpha))-m \alpha \ln (\lambda)+(\alpha-1) \sum_{i=1}^{m} \ln \left(x_{i}\right) \\
& -\frac{1}{\lambda} \sum_{i=1}^{m} x_{i}+\sum_{i=1}^{m} r_{i} \ln \left(1-\frac{\gamma\left(\alpha, \frac{x_{i}}{\lambda}\right)}{\Gamma(\alpha)}\right)
\end{aligned}
$$

Differentiating Equation (2.1) with respect to $\lambda$ and putting the derivative equal to zero we get

$$
\frac{-m \hat{\alpha}}{\hat{\lambda}}+\frac{m \bar{x}}{\hat{\lambda}^{2}}+\hbar\left(x_{i}, \hat{\alpha}, \hat{\lambda}\right)=0
$$


Where

$$
\hbar\left(x_{i}, \hat{\alpha}, \hat{\lambda}\right)=\frac{\partial \sum_{i=1}^{m} r_{i} \ln \left(1-\frac{\gamma\left(\hat{\alpha}, \frac{x_{i}}{\hat{\lambda}}\right)}{\Gamma(\hat{\alpha})}\right)}{\partial \hat{\lambda}}
$$

Differentiating Equation (2.1) with respect to $\alpha$ and putting the derivative equal to zero we get

$$
-n \psi(\hat{\alpha})-n \ln \hat{\lambda}+(\hat{\alpha}-1) \sum_{i=1}^{n} \ln \left(x_{i}\right)+\mathfrak{R}\left(x_{i}, \hat{\alpha}, \hat{\lambda}\right)=0
$$

Where $\frac{\partial \Gamma(\alpha)}{\partial \alpha}=\psi(\alpha), \psi(\alpha)$ is the digamma function .

And $\quad \mathfrak{R}\left(x_{i}, \hat{\alpha}, \hat{\lambda}\right)=\frac{\partial \sum_{i=1}^{m} r_{i} \ln \left(1-\frac{\gamma\left(\hat{\alpha}, \frac{x_{i}}{\hat{\lambda}}\right)}{\Gamma(\hat{\alpha})}\right)}{\partial \hat{\alpha}}$

Therefore $\hat{\alpha}$ and $\hat{\lambda}$ can be obtained as a solutions of Equations (2.2) and (2.3).

\subsection{Maximum Likelihood Estimation for Log-logistic Distribution}

The probability density function of the log-logistic distribution, denoted by $\operatorname{LL}(\varepsilon, \sigma)$, with scale parameter $\varepsilon>0$ and shape parameter $\sigma>0$ is given by

$$
f_{L L}(x ; \varepsilon, \sigma)=\frac{(\sigma / \varepsilon)(x / \varepsilon)^{\sigma-1}}{\left[1+(x / \varepsilon)^{\sigma}\right]^{2}}, \quad \quad \varepsilon, \sigma>0 \quad x>0 .
$$

Let $\mathrm{X}_{1: \mathrm{m}: \mathrm{n}}, \ldots, \mathrm{X}_{\mathrm{m}: \mathrm{m}: \mathrm{n}}$ be progressively type II censored sample from a two parameter loglogistic distribution, with censoring scheme $r=\left(r_{1}, \ldots, r_{m}\right)$. The likelihood function is given by

$$
L_{L L}(\varepsilon, \sigma)=k \prod_{i=1}^{m} f\left(x_{i: m: n}\right)\left[1-F\left(x_{i: m: n}\right)\right] r_{i}
$$

Where $k=n\left(n-1-r_{1}\right)\left(n-2-r_{1}-r_{2}\right) \ldots\left(n-m+1-r_{1}-\ldots-r_{m-1}\right)$.

For simplicity of notation, we will use $x_{i}$ instead of $x_{i: m: n}$.

$$
L_{L L}(\varepsilon, \sigma) \propto \frac{\sigma^{m}\left(\frac{1}{\varepsilon}\right)^{m \sigma} \prod_{i=1}^{m} x_{i}^{\sigma-1}}{\prod_{i=1}^{m}\left[1+\left(x_{i} / \varepsilon\right)^{\sigma}\right]^{2+r_{i}}}
$$


The logarithm of the likelihood function is given as

$\ln L_{L L}(\varepsilon, \sigma)=m \ln \sigma-m \sigma \ln \varepsilon+(\sigma-1) \sum_{i=1}^{m} \ln x_{i}-\sum_{i=1}^{m}\left(2+r_{i}\right) \ln \left[1+\left(x_{i} / \varepsilon\right)^{\sigma}\right]$

Differentiating Equation (2.4) with respect to $\varepsilon$ and putting the derivative equal to zero we get

$$
\begin{aligned}
& \frac{\partial L}{\partial \varepsilon}=\frac{-m \hat{\sigma}}{\hat{\varepsilon}}-\sum_{i=1}^{m}\left(2+r_{i}\right) \frac{\left(x_{i} / \hat{\varepsilon}\right)^{\hat{\sigma}}}{\left[1+\left(x_{i} / \hat{\varepsilon}\right)^{\hat{\sigma}}\right.} \frac{-\hat{\sigma}}{\hat{\varepsilon}}=0 \\
& m=\sum_{i=1}^{m}\left(2+r_{i}\right) \frac{\left(x_{i} / \hat{\varepsilon}\right)^{\hat{\sigma}}}{\left.1+\left(x_{i} / \hat{\varepsilon}\right)^{\hat{\sigma}}\right]} .
\end{aligned}
$$

Differentiating Equation (2.4) with respect to $\sigma$ and putting the derivative equal to zero we get

$$
\begin{aligned}
& \frac{\partial L}{\partial \sigma}=\frac{m}{\hat{\sigma}}-m \ln \hat{\varepsilon}+\sum_{i=1}^{m} \ln x_{i}-\sum_{i=1}^{m}\left(2+r_{i}\right) \frac{\left(x_{i} / \hat{\varepsilon}\right)^{\hat{\sigma}} \ln \left(x_{i} / \hat{\varepsilon}\right)}{\left[1+\left(x_{i} / \hat{\varepsilon}\right)^{\hat{\sigma}}\right]}=0 \\
& \frac{m}{\hat{\sigma}}-m \ln \hat{\varepsilon}+\sum_{i=1}^{m} \ln x_{i}-\sum_{i=1}^{m}\left(2+r_{i}\right) \frac{\left(x_{i} / \hat{\varepsilon}\right)^{\hat{\sigma}}}{\left[1+\left(x_{i} / \hat{\varepsilon}\right)^{\hat{\sigma}}\right]}\left(\ln x_{i}-\ln \hat{\varepsilon}\right)=0 \\
& \frac{m}{\hat{\sigma}}+\sum_{i=1}^{m} \ln x_{i}-\sum_{i=1}^{m}\left(2+r_{i}\right) \frac{\left(x_{i} / \hat{\varepsilon}\right)^{\hat{\sigma}} \ln x_{i}}{\left[1+\left(x_{i} / \hat{\varepsilon}\right)^{\hat{\sigma}}\right]}=0 .
\end{aligned}
$$

Therefore $\hat{\varepsilon}$ and $\hat{\sigma}$ can be obtained as a solutions of Equations (2.7) and (2.8).

\subsection{The Ratio of the Maximized Likelihood (RML)}

The ratio of the maximized likelihood (RML) is defined as

$$
\mathrm{RML}=L_{G A}(\hat{\lambda}, \hat{\alpha}) / L_{L L}(\hat{\varepsilon}, \hat{\sigma})
$$

Here $(\hat{\alpha}, \hat{\lambda})$ and $(\hat{\varepsilon}, \hat{\sigma})$ are the maximum likelihood estimators of $(\alpha, \lambda)$ and $(\varepsilon, \sigma)$ respectively. The test statistic T is the logarithm of RML and can be obtained as follows

$$
T=\ln R M L=\ln L_{G A}(\hat{\lambda}, \hat{\alpha})-\ln L_{L L}(\hat{\varepsilon}, \hat{\sigma})
$$

In this discrimination procedure, choose

(a) Gamma distribution if $\mathrm{T}>0$, i.e., if $\ln L_{G A}(\alpha, \lambda)>\ln L_{L L}(\hat{\varepsilon}, \hat{\sigma})$.

Or

(b) Log-logistic distribution if $\mathrm{T}<0$, i.e., if $\ln L_{L L}(\hat{\varepsilon}, \hat{\sigma})>\ln L_{G A}(\hat{\alpha}, \hat{\lambda})$. 


\subsection{Numerical Experiment}

In this section, the RML procedures are using for selecting between the Gamma and loglogistic distributions. A censoring scheme called progressive type II censoring is considered. The PCS's involved in the discrimination between the gamma and the loglogistic distributions based on likelihood ratio can be determined with more accuracy through simulated samples. For simplicities, the scale and shape parameters of the Gamma and log-logistic distributions can be fixed to some specific values without any loss of generality in assessing the relative performance of the RML procedure.

i. Firstly, when the true distribution is Gamma distribution computation of the PCS is performed as follows: By using the algorithm given by Balakrishna and Aggarwala (2000). The following steps are used to generate progressively Type-II right censored order statistics from Gamma distribution.

1. Generate $\mathrm{m}$ independent uniform $\mathrm{U}(0,1)$, random variables $\mathrm{W}_{1}, \mathrm{~W}_{2}, \ldots, \mathrm{W}_{\mathrm{m}}$.

2. For given values of the progressive censoring scheme $R_{1}, R_{2}, \ldots, R_{m}$. Set

$$
V_{i}=W_{i}^{1 /\left(i+\sum_{j=m-i+1}^{m} R_{j}\right)} \quad, i=1,2, \ldots, m
$$

3. Set $\mathrm{U}_{\mathrm{i}}=1-\mathrm{V}_{\mathrm{m}} \mathrm{V}_{\mathrm{m}-1} \ldots \mathrm{V}_{\mathrm{m}-\mathrm{i}+1}$ for $\mathrm{i}=1,2, \ldots, \mathrm{m}$. Then $\mathrm{U}_{1: \mathrm{m}: \mathrm{n}}, \mathrm{U}_{2: \mathrm{m}: \mathrm{n}}, \ldots ., \mathrm{U}_{\mathrm{m}: \mathrm{m}: \mathrm{n}}$ is $\mathrm{a}$ progressively Type-II right censored sample of size $\mathrm{m}$ from $\mathrm{U}(0,1)$.

4. For a given values of the two parameters

$$
T_{i}=\lambda G^{-1}\left(U_{i}\right), \quad, i=1,2, \ldots, m
$$

Where

$$
G(t)=\int_{0}^{t} \frac{1}{\Gamma \alpha} e^{-\frac{x_{i}}{\lambda}}\left(\frac{x_{i}}{\lambda}\right)^{\alpha-1}
$$

is a progressively Type-II right censored sample of size $m$ from the Gamma distribution.

5. After maximum likelihood estimation, both the Gamma and the log-logistic distributions have fitted to the sample, and a realization $t$ of the statistic $T=\ln \frac{L_{G A}}{L_{L L}}$, is calculated and stored.

6. Steps 4 and 5 are repeated many times (in this study, the repetition was done 100 times).

7. The approximate PCS under the assumption that the true distribution is Gamma is $\mathrm{PCS}_{\mathrm{GA}}=\operatorname{Pr}[\mathrm{T}>0] \approx$ (number of $\mathrm{t}$ values in step $5>0$ )/100. The result are given in Table1.

ii. Secondly, when the true distribution is log-logistic distribution computation of the PCS is performed as follows

1. The same steps in (1), (2), (3) are used. 
4. For a given values of the two parameters $\varepsilon$ and $\sigma$

$$
X_{i}=\varepsilon\left(\frac{u_{i}}{1-u_{i}}\right)^{1 / \sigma} \quad, i=1,2, \ldots, m
$$

is a progressively Type-II right censored sample of size $\mathrm{m}$ from the log-logistic dist.

5. After maximum likelihood estimation, both the Gamma and the log-logistic distributions have fitted to the sample, and a realization $t$ of the statistic $T=\ln \frac{L_{G A}}{L_{L L}}$ is calculated and stored.

6. Steps 4 and 5 are repeated many times (in this study, the repetition was done 100 times).

7. The approximate PCS under the assumption that the true distribution is loglogistic is $\mathrm{PCS}_{\mathrm{LL}}=\operatorname{Pr}[\mathrm{T}<0] \approx$ (number of $\mathrm{t}$ values in step $5<0$ )/100. The result is given in Table (2)

Table 1: PCS based on simulations when the data are from Gamma distribution

\begin{tabular}{|c|c|c|c|c|}
\hline $\mathrm{n}$ & $\mathrm{m}$ & censoring scheme $(\mathrm{R})$ & $\alpha$ & $\mathrm{PCS}_{\mathrm{GA}}$ \\
\hline \multirow[t]{5}{*}{25} & \multirow[t]{5}{*}{10} & \multirow[t]{5}{*}[\begin{array}{llllllllll}{4}&{0}&{0}&{2}&{0}&{0}&{4}&{2}&{0}&{3}\end{array}]{} & 1.2 & 0.72 \\
\hline & & & 1.5 & 0.74 \\
\hline & & & 2.2 & 0.77 \\
\hline & & & 2.5 & 0.81 \\
\hline & & & 3 & 0.84 \\
\hline \multirow[t]{5}{*}{20} & \multirow[t]{5}{*}{8} & \multirow[t]{5}{*}[\begin{array}{llllllll}{1}&{2}&{0}&{2}&{3}&{2}&{0}&{2}\end{array}]{} & 1.2 & 0.69 \\
\hline & & & 1.5 & 0.72 \\
\hline & & & 2.2 & 0.73 \\
\hline & & & 2.5 & 0.78 \\
\hline & & & 3 & 0.82 \\
\hline \multirow[t]{5}{*}{15} & \multirow[t]{5}{*}{6} & \multirow[t]{5}{*}[\begin{array}{llllll}{2}&{0}&{2}&{0}&{3}&{2}\end{array}]{} & 1.2 & 0.68 \\
\hline & & & 1.5 & 0.72 \\
\hline & & & 2.2 & 0.76 \\
\hline & & & 2.5 & 0.79 \\
\hline & & & 3 & 0.81 \\
\hline \multirow[t]{5}{*}{10} & \multirow[t]{5}{*}{4} & \multirow[t]{5}{*}[\begin{array}{llll}{2}&{0}&{2}&{2}\end{array}]{} & 1.2 & 0.65 \\
\hline & & & 1.5 & 0.69 \\
\hline & & & 2.2 & 0.71 \\
\hline & & & 2.5 & 0.74 \\
\hline & & & 3 & 0.78 \\
\hline
\end{tabular}


Table 2: PCS based on simulations when the data are from log-logistic distribution

\begin{tabular}{|c|c|c|c|c|}
\hline $\mathrm{n}$ & $\mathrm{m}$ & censoring scheme $(\mathrm{R})$ & $\sigma$ & $\mathrm{PCS}_{\mathrm{LL}}$ \\
\hline \multirow[t]{5}{*}{25} & \multirow[t]{5}{*}{10} & \multirow[t]{5}{*}[\begin{array}{llllllllll}{4}&{0}&{0}&{2}&{0}&{0}&{4}&{2}&{0}&{3}\end{array}]{} & 2.2 & 0.54 \\
\hline & & & 2.5 & 0.58 \\
\hline & & & 3 & 0.59 \\
\hline & & & 3.5 & 0.63 \\
\hline & & & 4 & 0.66 \\
\hline \multirow[t]{5}{*}{20} & \multirow[t]{5}{*}{8} & \multirow[t]{5}{*}[\begin{array}{llllllll}{1}&{2}&{0}&{2}&{3}&{2}&{0}&{2}\end{array}]{} & 2.2 & 0.51 \\
\hline & & & 2.5 & 0.56 \\
\hline & & & 3 & 0.60 \\
\hline & & & 3.5 & 0.61 \\
\hline & & & 4 & 0.64 \\
\hline \multirow[t]{5}{*}{15} & \multirow[t]{5}{*}{6} & \multirow[t]{5}{*}[\begin{array}{llllll}{2}&{0}&{2}&{0}&{3}&{2}\end{array}]{} & 2.2 & 0.49 \\
\hline & & & 2.5 & 0.51 \\
\hline & & & 3 & 0.52 \\
\hline & & & 3.5 & 0.57 \\
\hline & & & 4 & 0.60 \\
\hline \multirow[t]{5}{*}{10} & \multirow[t]{5}{*}{4} & \multirow[t]{5}{*}[\begin{array}{llll}{2}&{0}&{2}&{2}\end{array}]{} & 2.2 & 0.47 \\
\hline & & & 2.5 & 0.50 \\
\hline & & & 3 & 0.54 \\
\hline & & & 3.5 & 0.56 \\
\hline & & & 4 & 0.60 \\
\hline
\end{tabular}

\subsection{Data Analysis}

Nelson [1982, p.105] presented data on the time (in minutes) to breakdown of an insulating fluid in an accelerated test at 34 kilovolts. This data is given in Table 3.

Table 3 Nelson's Data

$\begin{array}{llllllllllll}0.19 & 0.78 & 0.96 & 1.31 & 2.78 & 3.16 & 4.15 & 4.67 & 4.85 & 6.50 & 7.35 & 8.01 \\ 8.27 & 12.06 & 31.75 & 32.52 & 33.91 & 36.71 & 72.89 & & & & & \end{array}$

Using Nelson data, the generation of progressively Type-II censored order statistics was illustrated by the example given in Balakrishnan and Aggarwala [2000]. Consider $m=8$ and the censoring scheme $\mathrm{R}=(0,0,3,0,3,0,0,5)$. The observations and censoring scheme are reported in Table 4.

Table 4: Progressively type II censored sample generated from the times to breakdown data

\begin{tabular}{|l|l|l|l|l|l|l|l|l|}
\hline $\mathrm{i}$ & 1 & 2 & 3 & 4 & 5 & 6 & 7 & 8 \\
\hline $\mathrm{x}_{\mathrm{i}}$ & 0.19 & 0.78 & 0.96 & 1.31 & 2.78 & 4.85 & 6.50 & 7.35 \\
\hline $\mathrm{Ln} \mathrm{x}_{\mathrm{i}}$ & -1.660 & -0.248 & -0.040 & 0.27 & 1.022 & 1.578 & 1.87 & 1.994 \\
\hline $\mathrm{r}_{\mathrm{i}}$ & 0 & 0 & 3 & 0 & 3 & 0 & 0 & 5 \\
\hline
\end{tabular}


From the formula described in (4.1,4.2), we obtain the MLEs of Gamma distribution $\hat{\lambda}$ $=0.67, \hat{\alpha}=0.54$, then $\ln \mathrm{L}_{\mathrm{GA}}=-36.8346$, Also we obtain the MLEs of log-logistic distribution to be $\hat{\varepsilon}=0.9027$ and $\hat{\sigma}=1.233$, then $\ln \mathrm{L}_{\mathrm{LL}}=-39.3475$.

Now using the following formula derived in (4.3) we calculate T.

$$
\begin{aligned}
& T=\ln R M L=\ln \frac{L_{G A}(\hat{\alpha}, \hat{\lambda})}{L_{L L}(\hat{\varepsilon}, \hat{\sigma})}=\ln L_{G A}(\hat{\alpha}, \hat{\lambda})-\ln L_{L L}(\hat{\varepsilon}, \hat{\sigma}) \\
& \mathrm{T}=2.5129
\end{aligned}
$$

Therefore, by using the RML test to discriminate between the two distributions in case of progressive censoring Type II, the gamma model is chosen for this data set.

\section{In Case of Complete Samples}

As special case of progressive censoring, the problem of discriminating between Gamma and log-logistic distribution functions in case of complete samples when censoring scheme $R_{i}=0$ is considered in this section.

\subsection{Likelihood Ratio Test}

Put $\mathrm{R}_{\mathrm{i}}=0$ in (2.1) and (2.4) we get the following log likelihood functions for complete samples

$$
\begin{aligned}
& \ln L_{G A}(\lambda, \alpha)=n \ln (\Gamma(\alpha))-n \alpha \ln (\lambda)+(\alpha-1) \sum_{i=1}^{n} \ln \left(x_{i}\right)-\frac{n \bar{x}}{\lambda} \\
& \ln L_{L L}(\varepsilon, \sigma)=n \ln \sigma-n \sigma \ln \varepsilon+(\sigma-1) \sum_{i=1}^{n} \ln x_{i}-2 \sum_{i=1}^{n} \ln \left[1+\left(x_{i} / \varepsilon\right)^{\sigma}\right]
\end{aligned}
$$

$\hat{\alpha}$ and $\hat{\lambda}$ can be obtained as a solutions of these two equations

$$
\begin{aligned}
& \hat{\lambda}-\frac{\bar{x}}{\hat{\alpha}}=0 \\
& -n \psi(\hat{\alpha})-n \ln (\bar{x})+n \ln (\hat{\alpha})+(\hat{\alpha}-1) \sum_{i=1}^{n} \ln \left(x_{i}\right)=0
\end{aligned}
$$

Similarly, $\hat{\varepsilon}$ and $\hat{\sigma}$ can be obtained as a solutions of these two equations

$$
\begin{aligned}
& n-2 \sum_{i=1}^{n} \frac{\left(x_{i} / \hat{\varepsilon}\right)^{\hat{\sigma}}}{\left[1+\left(x_{i} / \hat{\varepsilon}\right)^{\hat{\sigma}}\right]}=0 . \\
& \frac{n}{\hat{\sigma}}+\sum_{i=1}^{n} \ln x_{i}-2 \sum_{i=1}^{n} \frac{\left(x_{i} / \hat{\varepsilon}\right)^{\hat{\sigma}} \ln x_{i}}{\left[1+\left(x_{i} / \hat{\varepsilon}\right)^{\hat{\sigma}}\right]}=0 .
\end{aligned}
$$

The logarithm of RML can be obtained as follows

$$
T=\ln \frac{\ln L_{G A}(\hat{\lambda}, \hat{\alpha})}{L_{L L}(\hat{\varepsilon}, \hat{\sigma})}=\ln L_{G A}(\lambda, \alpha)-\ln L_{L L}(\hat{\varepsilon}, \hat{\sigma})
$$


Here $(\hat{\lambda}, \hat{\alpha})$ and $(\hat{\varepsilon}, \hat{\sigma})$ are the maximum likelihood estimators of $(\lambda, \alpha)$ and $(\varepsilon, \sigma)$ respectively.

In this discrimination procedure, choose

(a) Gamma distribution if $\mathrm{T}>0$, i.e., if $\ln L_{G A}(\alpha, \lambda)>\ln L_{L L}(\hat{\varepsilon}, \hat{\sigma})$.

Or

(b) Log-logistic distribution if $\mathrm{T}<0$, i.e., if $\ln L_{L L}(\hat{\varepsilon}, \hat{\sigma})>\ln L_{G A}(\hat{\alpha}, \hat{\lambda})$.

\subsection{Asymptotic Properties of the RML under Null Hypotheses}

In this section, the asymptotic distributions of the RML statistics will be obtained under null hypotheses in two different cases. From now on the almost sure convergence will be denoted by a.s.

For any Borel measurable function $h(),. \mathrm{E}_{\mathrm{GA}}(\mathrm{h}(\mathrm{U}))$ and $\mathrm{V}_{\mathrm{GA}}(\mathrm{h}(\mathrm{U}))$ denote mean and variance of $h(U)$ under the assumption that $U$ follows $G A(.,$.$) . Similarly define$ $\operatorname{ELL}(\mathrm{h}(\mathrm{U}))$ and $\operatorname{VLL}(\mathrm{h}(\mathrm{U}))$ as mean and variance of $\mathrm{h}(\mathrm{U})$ under the assumption that $\mathrm{U}$ follows $\mathrm{LL}(.,$.$) . Also if \mathrm{g}($.) and $\mathrm{h}($.) are two Borel measurable functions, define along the same line

$\operatorname{Cov}_{\mathrm{gA}}(\mathrm{g}(\mathrm{U}), \mathrm{h}(\mathrm{U}))=\mathrm{E}_{\mathrm{GA}}(\mathrm{g}(\mathrm{U}) \cdot \mathrm{h}(\mathrm{U}))-\mathrm{E}_{\mathrm{GA}} \mathrm{g}(\mathrm{U}) \cdot \mathrm{E}_{\mathrm{GA}} \mathrm{h}(\mathrm{U})$, and similarly

$\operatorname{Cov}_{L L}(g(U), h(U))=E_{L L}(g(U) h(U))-E_{L L} g(U) \cdot E_{L L} h(U)$,

Lemma 1. Under the assumption that the data are from $\operatorname{GA}(\alpha, \lambda)$, we have the following results as $\mathrm{n} \longrightarrow \infty$

a. $\hat{\alpha} \longrightarrow \alpha, \hat{\lambda} \longrightarrow \lambda$ where

$$
E_{G A}\left(\ln f_{G A}(X ; \lambda, \alpha)\right)=\max _{\bar{\alpha}, \bar{\lambda}} E_{G A}\left(\ln f_{G A}(X ; \bar{\lambda}, \bar{\alpha})\right)
$$

b. $\hat{\sigma} \longrightarrow \tilde{\sigma}, \hat{\varepsilon} \longrightarrow \tilde{\varepsilon}$, a.s, where

$$
E_{G A}\left(\ln f_{L L}(X ; \tilde{\varepsilon}, \tilde{\sigma})\right)=\max _{\sigma, \varepsilon} E_{G A}\left(\ln f_{L L}(X ; \varepsilon, \sigma)\right)
$$

Denote

$$
T^{*}=\ln \left[\frac{L_{G A}(\lambda, \alpha)}{L_{L L}(\tilde{\varepsilon}, \tilde{\sigma})}\right]
$$


c. $\quad \frac{1}{\sqrt{n}}\left[T-E_{G A}(T)\right]$ Asymptotically equivalent to $\frac{1}{\sqrt{n}}\left[T^{*}-E_{G A}\left(T^{*}\right)\right]$

Proof of Lemma 1. The proof follows using similar arguments as of White (1982.theorem 1) and therefore it is omitted.

Lemma 2. Under the assumption that the data are from $\operatorname{LL}(\varepsilon, \sigma)$, we have the following results as $\mathrm{n} \longrightarrow \infty$

a. $\hat{\sigma} \longrightarrow \sigma, \hat{\varepsilon} \longrightarrow \varepsilon$ where

$$
E_{L L}\left(\ln f_{L L}(X ; \varepsilon, \sigma)\right)=\max _{\bar{\sigma}, \bar{\varepsilon}} E_{L L}\left(\ln f_{L L}(X ; \bar{\varepsilon}, \bar{\sigma})\right)
$$

b. $\hat{\alpha} \longrightarrow \tilde{\alpha}, \hat{\lambda} \longrightarrow \tilde{\lambda}$, a.s, where

$$
E_{L L}\left(\ln f_{G A}(X ; \tilde{\lambda}, \tilde{\alpha})\right)=\max _{\alpha, \lambda} E_{L L}\left(\ln f_{G A}(X ; \lambda, \alpha)\right)
$$

Denote

$$
T^{*}=\ln \left[\frac{L_{G A}(\tilde{\lambda}, \tilde{\alpha})}{L_{L L}(\varepsilon, \sigma)}\right]
$$

c. $\quad \frac{1}{\sqrt{n}}\left[T-E_{L L}(T)\right]$ Asymptotically equivalent to $\frac{1}{\sqrt{n}}\left[T^{*}-E_{L L}\left(T^{*}\right)\right]$

Theorem 1. Under the assumption that the data are from a Gamma distribution, the distribution of $\mathrm{T}$ is approximately normally distributed with mean $\mathrm{E}_{\mathrm{GA}}(\mathrm{T})$ and variance $\mathrm{V}_{\mathrm{GA}}(\mathrm{T})$

Proof: Using the central limit theorem and using (iii) of Lemma 1, one can easily shows that $\frac{1}{\sqrt{n}}\left[T^{*}-E_{G A}\left(T^{*}\right)\right]$ is asymptotically normally distributed.

Theorem 2. Under the assumption that the data are from log-logistic distribution, the distribution of $\mathrm{T}$ is approximately normally distributed with mean $\mathrm{E}_{\mathrm{LL}}(\mathrm{T})$ and variance $\mathrm{V}_{\mathrm{LL}}(\mathrm{T})$. The proof follows along the same line as of Theorem 1.

Case (1): The data are coming from a gamma distribution and the alternative is a loglogistic distribution.

Assumed that $n$ data points $\mathrm{x}_{1}, \mathrm{x}_{2}, \ldots, \mathrm{x}_{\mathrm{n}}$ are obtained from $\mathrm{GA}(\lambda, \alpha)$ with scale parameter $\lambda$ and shape parameter $\alpha$. 
Now to obtain $\tilde{\varepsilon}$ and $\tilde{\sigma}$ as defined in Lemma 1 , let us define

$$
\begin{aligned}
h(\varepsilon, \sigma) & =E_{G A}\left[\ln \left(f_{L L}(X ; \varepsilon, \sigma)\right]\right. \\
& =E_{G A}\left[\ln \sigma-\ln \varepsilon+(\sigma-1)[\ln X-\ln \varepsilon]-2 \ln \left[1+\left(\frac{X}{\varepsilon}\right)^{\sigma}\right]\right] \\
& =\ln (\sigma)+(\sigma-1)\left[E_{G A} \ln (y)+\ln (\lambda)\right]-\sigma \ln \varepsilon-2 E_{G A}\left(\ln \left[1+\left(\frac{\lambda y}{\varepsilon}\right)^{\sigma}\right]\right)
\end{aligned}
$$

Where $\mathrm{y} \sim$ gamma distribution $(1, \alpha)$

Differentiating Equation (3.2) with respect to $\varepsilon, \sigma$ and equating to zero. Therefore, $\varepsilon$ and $\tilde{\sigma}$ can be obtained as solutions of the following equations

$$
\begin{aligned}
& \tilde{\varepsilon}+\frac{\tilde{\sigma}}{2\left[\partial E_{G A}\left(\ln \left[1+\left(\frac{\lambda y}{\varepsilon}\right)^{\sigma}\right]\right) / \partial \varepsilon\right]}=0 \\
& \frac{1}{\tilde{\sigma}}+\left[E_{G A} \ln (y)+\ln (\lambda)\right]-\ln \tilde{\varepsilon}-2 \frac{\partial E_{G A} \ln \left[1+\left(\frac{\lambda y}{\varepsilon}\right)^{\sigma}\right]}{\partial \varepsilon}=0
\end{aligned}
$$

From these equations it is clear that $\tilde{\varepsilon}$ and $\tilde{\sigma}$ are both functions of $\lambda$ and $\alpha$.

To obtain $E_{G A}(T)$ and $V_{G A}(T)$ denote,

$$
A M_{L L}=\lim _{n \rightarrow \infty} \frac{1}{n} E_{L L}(T) \text { and } A V_{L L}=\lim _{n \rightarrow \infty} \frac{1}{n} V_{L L}(T),
$$

Therefore for large $n$ we have,

$$
\begin{gathered}
\lim _{n \rightarrow \infty} \frac{1}{n} E_{G A}(T)=A M_{G A}=E_{G A}\left[\ln f_{G A}(X ; \lambda, \alpha)-\ln f_{L L}(X ; \tilde{\varepsilon}, \tilde{\sigma})\right] \\
A M_{G A}=-\alpha \ln \lambda-\ln \Gamma(\alpha)+(\alpha-\sigma)\left(E_{G A} \ln (y)+\ln (\lambda)\right)-\frac{\lambda \alpha}{\lambda}-\ln (\sigma)-\sigma \ln \varepsilon-2 E_{G A}\left(\ln \left[1+\left(\frac{\lambda y}{\varepsilon}\right)^{\sigma}\right]\right) \\
\lim _{n \rightarrow \infty} \frac{1}{n} V_{G A}(T)=A V_{G A}=V_{G A}\left[\ln f_{G A}(X ; \lambda, \alpha)-\ln f_{L L}(X ; \tilde{\varepsilon}, \tilde{\sigma})\right] \\
A V_{G A}=(\alpha-\sigma)^{2} \psi_{1}(\alpha)+\alpha+4 V_{G A}\left(\ln \left[1+\left(\frac{\lambda y}{\varepsilon}\right)^{\sigma}\right]\right)-2(\alpha-\sigma) \operatorname{cov}(\ln (\lambda y), y) \\
+4(\alpha-\sigma) \operatorname{cov}\left(\ln (\lambda y), \ln \left[1+\left(\frac{\lambda y}{\varepsilon}\right)^{\sigma}\right]\right)-4 \operatorname{cov}\left(y, \ln \left[1+\left(\frac{\lambda y}{\varepsilon}\right)^{\sigma}\right]\right)
\end{gathered}
$$


Case (2): The data are coming from a log-logistic distribution and the alternative is a Gamma distribution.

Assumed that $\mathrm{n}$ data points $\mathrm{x}_{1}, \mathrm{x}_{2}, \ldots, \mathrm{x}_{\mathrm{n}}$ are obtained from $\operatorname{LL}(\varepsilon, \sigma)$ with scale parameter $\varepsilon$ and shape parameter $\sigma$. Now to obtain $\tilde{\lambda}, \tilde{\alpha}$ let us define

$$
\begin{aligned}
\mathrm{g}(\lambda, \alpha) & =E_{L L}\left[\ln f_{G A}(x ; \lambda, \alpha)\right] \\
& =-\alpha \ln \lambda-\ln \Gamma \alpha+(\alpha-1) E_{L L}(\ln (x))-\frac{1}{\lambda} E_{L L}(x) \\
g(\lambda, \alpha) & =-\alpha \ln \lambda-\ln \Gamma \alpha+(\alpha-1)(E(\ln y)+\ln \varepsilon)-\frac{1}{\lambda} \frac{\varepsilon \pi / \sigma}{\sin (\pi / \sigma)}
\end{aligned}
$$

If $\sigma>1$ else undefined, Where $y \sim L L(1,1)$

Differentiating Equation (3.7) with respect to $\alpha, \lambda$ and equating to zero. Therefore, $\tilde{\lambda}$ and $\alpha$ can be obtained as solutions of the following equations

$$
\begin{aligned}
& -\ln \tilde{\lambda}-\psi(\alpha)+E_{L L}(y)+\ln \varepsilon=0 \\
& \frac{\tilde{\alpha}}{\tilde{\lambda}}+\frac{1}{\tilde{\lambda}^{2}} \frac{\varepsilon \pi / \sigma}{\sin (\pi / \sigma)}=0
\end{aligned}
$$

From these equations it is clear that $\tilde{\lambda}$ and $\tilde{\alpha}$ are both functions of $\varepsilon$ and $\sigma$. Now to obtain $E_{L L}(T)$ and $V_{L L}(T)$, denote

$$
\begin{aligned}
& A M_{L L}=\lim _{n \rightarrow \infty} \frac{1}{n} E_{L L}(T) \text { and } A V_{L L}=\lim _{n \rightarrow \infty} \frac{1}{n} V_{L L}(T), \\
& \lim _{n \rightarrow \infty} \frac{1}{n} E_{L L}(T)=A M_{L L}=E_{L L}\left[\ln f_{G A}(X ; \tilde{\lambda}, \tilde{\alpha})-\ln f_{L L}(X ; \varepsilon, \sigma)\right] \\
& =E_{L L}\left(\ln f_{G A}(X ; \tilde{\lambda}, \tilde{\alpha})\right)-E_{L L}\left[\ln f_{L L}(X ; \varepsilon, \sigma)\right] \\
& A M_{L L}=-\alpha \ln \lambda-\ln \Gamma \alpha-\frac{1}{\lambda} \frac{\varepsilon \pi / \sigma}{\sin (\pi / \sigma)}+(\alpha-\sigma)(E(\ln y)+\ln \varepsilon)-\ln (\sigma) \\
& +\sigma \ln \varepsilon+2 E_{L L}(\ln (1+z)) \quad \text { If } \sigma>1 \text { else undefined }
\end{aligned}
$$

Where $y \sim L \mathrm{~L}(1, \sigma)$ and $z \sim L \mathrm{~L}(1,1)$. Moreover;

$$
\lim _{n \rightarrow \infty} \frac{1}{n} V_{L L}(T)=A V_{L L}=V_{L L}\left[\ln f_{G A}(X ; \lambda, \alpha)-\ln f_{L L}(X ; \tilde{\varepsilon} \tilde{\sim} \tilde{\sigma})\right]
$$




$$
\begin{gathered}
A V_{L L}=(\alpha-\sigma){ }^{2} V_{L L}(\ln y)+\frac{1}{\lambda^{2}}\left(\varepsilon^{2}\left(2 b / \sin 2 b-b^{2} / \sin b^{2}\right)\right)+4 V_{L L}\left(\left[1+y^{\sigma}\right]\right) \\
\left.-\frac{2 \varepsilon(\alpha-\sigma)}{\lambda} \operatorname{cov}((\ln y), y)\right)+4(\alpha-\sigma) \operatorname{cov}\left((\ln y), \ln \left[1+y^{\sigma}\right]\right)-\frac{4 \varepsilon}{\lambda} \operatorname{cov}\left(\ln \left(1+y^{\sigma}\right), y\right)
\end{gathered}
$$

Where $y \sim L \mathrm{~L}(1, \sigma)$

Different values of $\tilde{\sigma}, \tilde{\varepsilon}, A M_{G A}(\lambda, \alpha), A V_{G A}(\lambda, \alpha), \tilde{\alpha}$ and $\tilde{\lambda}, A M_{L L}(\varepsilon, \sigma) A V_{L L}(\varepsilon, \sigma)$ are computed numerically by using Equations (3.3), (3.4), (3.5), (3.6), (3.8), (3.9), (3.10),(3.11) and reported in Table 5, Table 6.

Table 5: Different Values of $\operatorname{AM}_{\mathrm{GA}}(\alpha), \mathbf{A V}_{\mathrm{GA}}(\alpha), \tilde{\sigma}$ and $\tilde{\varepsilon}$ for different $\alpha$ when $\lambda=1$

\begin{tabular}{lllll}
\hline$\alpha$ & $\mathrm{AM}_{\mathrm{GA}}$ & $\mathrm{AV}_{\mathrm{GA}}$ & $\tilde{\sigma}$ & $\tilde{\varepsilon}$ \\
\hline 1.2 & -0.179 & 0.476 & 0.161 & 375.432 \\
1.5 & -0.102 & 0.472 & 0.168 & 394.131 \\
2 & 0.027 & 0.447 & 0.178 & 424.43 \\
2.2 & 0.076 & 0.428 & 0.181 & 429.509 \\
2.5 & 0.076 & 0.428 & 0.186 & 431.661 \\
3 & 0.076 & 0.428 & 0.192 & 457.002 \\
\hline
\end{tabular}

Table 6 Different Values of $\operatorname{AM}_{L L}(\sigma), \operatorname{AV}_{L L}(\sigma), \tilde{\lambda}$ and $\tilde{\alpha}$ for different $\sigma$ when $\varepsilon=1$

\begin{tabular}{lllll}
\hline$\sigma$ & $\mathrm{AM}_{\mathrm{LL}}$ & $\mathrm{AV}_{\mathrm{LL}}$ & $\tilde{\lambda}$ & $\tilde{\alpha}$ \\
\hline 2.2 & -0.109 & 5.397 & 0.956 & 1.509 \\
2.5 & -0.087 & 2.326 & 0.679 & 1.945 \\
3 & -0.064 & 2.058 & 0.434 & 2.788 \\
3.2 & -0.058 & 2.253 & 0.373 & 3.166 \\
3.5 & -0.051 & 2.681 & 0.304 & 3.78 \\
4 & -0.042 & 3.635 & 0.226 & 4.922 \\
\hline
\end{tabular}

\subsection{Determination of Sample Size}

In this Section, we propose a method to determine the minimum sample size needed to discriminate between Gamma and log-logistic distributions, for a given user specified probability of correct selection (PCS). Intuitively, it is clear that if two distributions are very close, one needs a very large sample size to discriminate between them for a given probability of correct selection. On the other hand if two distribution functions are quite different, then one may not need very large sample size to discriminate between two distribution functions. It is also true that if two distribution functions are very close to each other, then one may not need to differentiate the two distributions. 
It is expected that the user will specify beforehand the PCS and tolerance limit in terms of the distance between two distribution functions in terms of the distance between two distribution functions. Based on the PCS, the required minimum sample size can be determined. The tolerance limit simply indicates that the user does not want to make the distinction between two distribution functions if their distance is less than the tolerance limit. Based on the PCS and the tolerance limit, the required minimum sample size can be determined.

In section 3.2 the RML statistics follow normal distribution approximately for large $n$ will be observed. Now it will be used with the help of $\mathrm{K}-\mathrm{S}$ distance to determine the required sample size $n$.

Using Theorem 1 and since it is assumed that the data are coming from $\operatorname{GA}(\lambda, \alpha)$. In this case the probability of correct selection $\mathrm{PCS}_{\mathrm{GA}}$ is given by

$$
\begin{aligned}
\operatorname{PCS}_{\mathrm{GA}}(\lambda, \alpha) & =\operatorname{Pr}(\mathrm{T}>0) \\
& \approx 1-\Phi\left(-\frac{E_{G A}(T)}{\sqrt{V_{G A}(T)}}\right) \\
& =\Phi\left(\frac{n \times A M_{G A}(\lambda, \alpha)}{\sqrt{n \times A V_{G A}(\lambda, \alpha)}}\right) .
\end{aligned}
$$

Also using Theorem 2 and since it is assumed that the data are coming from $\operatorname{LL}(\varepsilon, \sigma)$. In this case, the $\mathrm{PCS}_{\mathrm{LL}}$ is given by

$$
\begin{aligned}
\operatorname{PCS}_{L L}(\varepsilon, \sigma)= & \operatorname{Pr}(\mathrm{T}<0) \\
& =1-\Phi\left(\frac{E_{L L}(T)}{\sqrt{V_{L L}(T)}}\right) \\
& =1-\Phi\left(\frac{n \times A M_{L L}(\varepsilon, \sigma)}{\sqrt{n \times A V_{L L}(\varepsilon, \sigma)}}\right) .
\end{aligned}
$$

Where AM and AV denote the asymptotic mean and variance respectively, and $\Phi$ is the distribution function of the standard normal random variable.

Therefore, to determine the minimum sample size required to achieve at least $\alpha^{*}$ protection level, equate $\operatorname{PCS}_{\mathrm{GA}}(\lambda, \alpha)$ by $\operatorname{PCS}_{\mathrm{LL}}(\varepsilon, \sigma)$ i.e., Let

$$
\Phi\left(\frac{n \times A M_{G A}(\lambda, \alpha)}{\sqrt{n \times A V_{W E}(\lambda, \alpha)}}\right)=\alpha^{*} \quad \text { and } \quad \Phi\left(-\frac{n \times A M_{L L}(\varepsilon, \sigma)}{\sqrt{n \times A V_{L L}(\varepsilon, \sigma)}}\right)=\alpha^{*}
$$


From previous equations give $\mathrm{n}_{1}$ and $\mathrm{n}_{2}$ as

$$
n_{1}=\frac{Z_{\alpha}{ }^{2} \times A V_{G A}(\lambda, \alpha)}{\left(A M_{G A}(\lambda, \alpha)\right)^{2}} \quad \text { (3.12), } \quad n_{2}=\frac{Z_{\alpha}{ }^{2} \times A V_{L L}(\varepsilon, \sigma)}{\left(A M_{L L}(\varepsilon, \sigma)\right)^{2}}
$$

Here $\mathrm{z}_{\alpha}$ is the $\alpha$-th percentile point of a standard normal distribution. reported $\mathrm{n}_{1}$, with the help of Table(5) for different $\alpha$ when the protection level $\alpha=0.7$ in Table(7), reported $\mathrm{n}_{2}$, with the help of Table(6) for different $\sigma$ when the protection level $\alpha=0.7$, in Table(8).

Therefore, the minimum sample size $\mathrm{n}$ required to discriminate between Gamma and loglogistic distribution can be taken as $\max \left(\mathrm{n}_{1} ; \mathrm{n}_{2}\right)$.

The distance between two distribution functions is defined by the $\mathrm{K}-\mathrm{S}$ distance. The $\mathrm{K}-\mathrm{S}$ distance between two distribution functions, say $\mathrm{F}(\mathrm{x})$ and $\mathrm{G}(\mathrm{x})$ is defined as

$$
\sup _{x}|F(x)-G(x)|
$$

K-S distance between $\operatorname{GA}(1, \alpha)$ and $\operatorname{LL}(\tilde{\varepsilon}, \tilde{\sigma})$ reported with the help of Table(5) for different values of $\alpha$, in Table(7). Similarly K-S distance between $\operatorname{LL}(1, \sigma)$ and $\operatorname{GA}(\tilde{\lambda}$, $\tilde{\alpha})$ reported with the help of Table(6) for different values of $\sigma$, in Table(8).

If one knows the range of the shape parameter of the null distribution and for a given PCS that achieves a certain protection level $\mathrm{P}^{*}$, then the minimum sample size can be obtained by taking the maximum n obtained from Equations (3.12) and (3.13).

But unfortunately in practice the shape parameter may be completely unknown; therefore, the K-S distances can replace the unknown parameters in taking the decision. That is, for a given protection level $\mathrm{P}^{*}$ and a given pre-specified tolerance limit $\mathrm{D}^{*}$, the minimum sample size can be obtained by taking the maximum $\mathrm{n}$ obtained from Equations (3.12) and (3.13). For example, suppose that for a given $\mathrm{P}^{*}=0.7$ and for $\alpha=2.2$ and $\sigma=2.2$, then from Tables 7 and 8 the minimum sample size required to discriminate between Weibull and log-logistic distributions is $\max (21,125)=125$.

On the other hand if and $\sigma$ are unknown and suppose that the practitioner wants to discriminate between Gamma and log-logistic distribution functions only when the distance between them is greater than or equal 0.217 , i.e., $\mathrm{D}^{*} \geq 0.217$ and with $\mathrm{P}^{*}=0.7$. Then from Tables 7 and 8 , it is clear that, $\mathrm{D}^{*} \geq 0.217$ if $\geq 1.2$ and $\sigma \geq 2.2$. Also, when the null distribution is Gamma, then for the tolerance limit $\mathrm{D}^{*} \geq 0.217$, one needs $\mathrm{n}=5$ to meet the PCS, $\mathrm{P}^{*}=0.7$. Similarly when the null distribution is log-logistic then one needs $\mathrm{n}=125$ to meet the same protection level. Finally, the minimum sample size required to discriminate between Gamma and log-logistic distributions with $\mathrm{P}^{*}=0.7$ and $\mathrm{D}^{*} \geq 0.217$ is $\max (5,125)=125$. 
Table 7: $\quad$ The minimum sample size $\mathbf{n}$ for $\mathbf{P}^{*}=0.7$, when the null distribution is gamma distribution is presented. The $\mathrm{K}-\mathrm{S}$ distance between $\mathrm{GA}(1, \alpha)$

and $\mathbf{L L}(\tilde{\varepsilon}, \tilde{\sigma})$ for different values of $\alpha$ is reported

\begin{tabular}{lcccccc}
\hline$\alpha$ & 1.2 & 1.5 & 2 & 2.2 & 2.5 & 3 \\
$\mathrm{n}$ & 5 & 13 & 169 & 21 & 21 & 21 \\
$\mathrm{~K}-\mathrm{S}$ & 0.217 & 0.230 & 0.230 & 0.227 & 0.227 & 0.227 \\
\hline
\end{tabular}

Table 8: $\quad$ The minimum sample size $n$ for $P^{*}=0.7$, when the null distribution is log-logistic distribution is presented. The K-S distance between LL(1, $\sigma)$ and $\operatorname{GA}(\tilde{\lambda}, \tilde{\alpha})$ for different values of $\sigma$ is reported.

\begin{tabular}{lcccccc}
\hline$\sigma$ & 2.2 & 2.5 & 3 & 3.2 & 3.5 & 4 \\
$\mathrm{n}$ & 125 & 85 & 137 & 183 & 283 & 565 \\
$\mathrm{~K}-\mathrm{S}$ & 0.351 & 0.310 & 0.265 & 0.240 & 0.232 & 0.220 \\
\hline
\end{tabular}

Notice that, Tables 7 and 8 are obtained for the protection level 0.7 but for other protection levels the tables can be easily modified. For example, if we need a sample size corresponding to protection level $\mathrm{P}^{*}=0.9$, then all the entries corresponding to the row of n, must be multiplied by $Z_{0.9}^{2} / Z_{0.7}^{2}$.

\subsection{Numerical Experiments}

In this section some experimental results is presented to examine how the asymptotic results derived in Section 3.2 behave for finite sample sizes. Moreover when the sample size is not sufficiently large, the PCS's involved in the discrimination between the gamma and the log-logistic distributions based on likelihood ratio can be determined with more accuracy through MC simulations sample. The PCSs obtained using simulations and based on the asymptotic results derived in Section 3.2 are compared.

Different sample sizes and different shape parameters of the null distributions are considered. The details are explained below. First case when the null distribution is gamma and the alternative is log-logistic. In this case consider $n=20,40,60,80,100$ and $\alpha=1.2,1.5,2,2.2,2.5$ and 3. Computation of the PCS is performed as follows

1. For a sample size $\mathrm{n}$, a random sample $\left\{\mathrm{x}_{1}, \mathrm{x}_{2}, \ldots \ldots, \mathrm{x}_{\mathrm{n}}\right\}$ is generated from a $\mathrm{GA}(1, \alpha)$ distribution. 
2. By maximum likelihood, both the gamma and the log-logistic distributions are fitted to the sample $\left\{\mathrm{x}_{1}, \mathrm{x}_{2}, \ldots \ldots, \mathrm{x}_{\mathrm{n}}\right\}$, and a realization, $\mathrm{t}$, of the statistic $T=\ln \frac{L_{G A}}{L_{L L}}$, is calculated and stored.

3. Steps 1 and 2 are repeated many times (in this study, the repetition was done 10,000 times).

4. The approximate PCS under the assumption that the true distribution is gamma, is: $\mathrm{PCS}_{\mathrm{GA}}=\operatorname{Pr}[\mathrm{T}>0] \approx$ (number of $\mathrm{t}$ values in step $2>0$ ) $/ 10,000$.

Also the PCS's obtained by using the asymptotic results as given in section 3.2 are computed. The results are reported in Table 9 in section 3.5.

Similarly, the results when the null distribution is log-logistic and the alternative is gamma are obtained. In this case considered the same set of $\mathrm{n}$ and $\sigma=2.2,2.5,3,3.2,3.5$ and 4. The results are reported in Table 10 in section 3.5.

\subsection{PCS of RMKLD}

In probability and information theory, the Kullback-Leibler divergence (also information discrepancy, information gain, relative entropy, or KLD) is a non-symmetric measure of the difference (dissimilarity) between two probability distributions $f_{1}(x)$ and $f_{2}(x)$ it denotes the "information lost when $f_{2}(x)$ is used to approximate $f_{1}(x)$ or the distance from $f_{1}(x)$ to $f_{2}(x)$ ". In other words, KLD is a measure of inefficiency of assuming that the distribution is $f_{2}(x)$ when the true distribution is $f_{1}(x)$. Since the measure from $f_{1}(x)$ to $f_{2}(x)$ is not the same as the measure from $f_{2}(x)$ to $f_{1}(x)$, then it can be conceptualized as a "directed/oriented distance" between the two models.

The KLD is a natural distance function between models. It is usually used as a logical basis for model selection in conjunction with likelihood inference. Values of KLD are not based on only the mean and variance of the distributions; rather, the distributions in their entirety are the subject of comparison. The later is regarded an advantage of the KLD as a test statistic. The KLD is defined as follow

$$
\begin{aligned}
K L D(h(x), f(x)) & =\int_{0}^{\infty} f_{1}(x) \ln \left(\frac{f_{1}(x)}{f_{2}(x)}\right) d x \\
& =\int_{0}^{\infty} f_{1}(x) \ln \left(f_{1}(x)\right) d x-\int_{0}^{\infty} f_{1}(x) \ln \left(f_{2}(x)\right) d x
\end{aligned}
$$


It is well known that

1. $\quad \operatorname{KLD}\left(f_{1}(x), f_{2}(x)\right) \neq \operatorname{KLD}\left(f_{2}(x), f_{1}(x)\right)$

2. $\operatorname{KLD}\left(f_{1}(x), f_{2}(x)\right) \geq 0$ and the equality holds if and only if $f_{1}(x)=f_{2}(x)$.

The smaller $\operatorname{KLD}\left(f_{1}(x), f_{2}(x)\right)$ means that " $f_{1}(x)$ " is preferred and large values of KLD favor " $f_{2}(x)$ ". However, the KLD (based test statistic) is considered as a ruler to measure the similarity between the two hypotheses / distributions.

The test statistic is defined as the natural logarithm of two ratios of KLDs. The idea is similar to RML, in which our interested to select a model maximizing the likelihood. But in KLD method our interested to select a model minimizing the KLD, That's why it named as ratio of minimized KLD (RMKLD) which is defined as follow

$$
R M K L D=\ln \left(\frac{K L D\left(f_{1}(x), f_{2}(x)\right.}{K L D\left(f_{2}(x), f_{1}(x)\right.}\right)
$$

In this Section how the proposed RMKLD test statistic work for different parameters and sample sizes is presented.

First considered case 1 when the null distribution is gamma. The RMKLD testing procedure is introduced as follows

1. For a sample size $\mathrm{n}$, a random sample $\left\{\mathrm{x}_{1}, \mathrm{x}_{2}, \ldots \ldots, \mathrm{x}_{\mathrm{n}}\right\}$ is generated from a $\mathrm{GA}(1, \alpha)$ distribution.

2. Calculate $K L D\left(f_{1}(x), f_{2}(x)\right.$ and $K L D\left(f_{2}(x), f_{1}(x)\right.$.

$$
R M K L D=\ln \left(\frac{K L D\left(f_{1}(x), f_{2}(x)\right.}{K L D\left(f_{2}(x), f_{1}(x)\right.}\right)
$$

is calculated and stored.

3. Select $f_{1}(x)$ if KLD $<0$.

4. Steps 2 and 3 are repeated many times (in this study, the repetition was done 10,000 times).

5. Compute the percentage of the times if RMKLD $<0$ as the PCS of gamma distribution.

$\mathrm{PCS}_{\mathrm{GA}}=($ number of RMKLD values in step $2<0) / 10,000$

The results are reported in Table 9.

Similarly, the $\mathrm{PCS}_{\mathrm{LL}}$ when the null distribution is log-logistic and the alternative is gamma are obtained. Results are reported in Table 10. 
Table 9: The Probability of Correct Selection Based on Simulations and also Based on Asymptotic Results When the Null Distribution is Gamma distribution

\begin{tabular}{|c|c|c|c|c|c|c|}
\hline \multirow[b]{2}{*}{$\alpha$} & & \multicolumn{5}{|c|}{$\mathrm{n}$} \\
\hline & PCS & 20 & 40 & 60 & 80 & 100 \\
\hline \multirow[t]{3}{*}{1.2} & RMLS & 0.77 & 0.83 & 0.89 & 0.91 & 0.92 \\
\hline & RMLAR & $(0.75)$ & $(0.77)$ & $(0.78)$ & $(0.86)$ & $(0.89)$ \\
\hline & RMKLD & 0.83 & 0.86 & 0.92 & 0.94 & 0.95 \\
\hline \multirow[t]{3}{*}{1.5} & RMLS & 0.79 & 0.84 & 0.88 & 0.90 & 0.93 \\
\hline & RMLAR & $(0.76)$ & $(0.80)$ & $(0.83)$ & $(0.85)$ & $(0.90)$ \\
\hline & RMKLD & 0.85 & 0.87 & 0.92 & 0.93 & 0.96 \\
\hline \multirow[t]{3}{*}{2.0} & RMLS & 0.80 & 0.84 & 0.89 & 0.92 & 0.93 \\
\hline & RMLAR & $(0.76)$ & $(0.79)$ & $(0.83)$ & $(0.86)$ & $(0.92)$ \\
\hline & RMKLD & 0.85 & 0.88 & 0.92 & 0.95 & 0.96 \\
\hline \multirow[t]{3}{*}{2.2} & RMLS & 0.82 & 0.86 & 0.90 & 0.91 & 0.94 \\
\hline & RMLAR & $(0.78)$ & $(0.82)$ & $(0.84)$ & $(0.88)$ & $(0.93)$ \\
\hline & RMKLD & 0.86 & 0.89 & 0.94 & 0.96 & 0.97 \\
\hline \multirow[t]{3}{*}{2.5} & RMLS & 0.83 & 0.86 & 0.91 & 0.93 & 0.94 \\
\hline & RMLAR & $(0.80)$ & $(0.83)$ & $(0.86)$ & $(0.92)$ & $(0.93)$ \\
\hline & RMKLD & 0.88 & 0.90 & 0.95 & 0.96 & 0.98 \\
\hline \multirow[t]{3}{*}{3.00} & RMLS & 0.85 & 0.89 & 0.93 & 0.94 & 0.95 \\
\hline & RMLAR & $(0.83)$ & $(0.86)$ & $(0.90)$ & $(0.91)$ & $(0.94)$ \\
\hline & RMKLD & 0.90 & 0.92 & 0.96 & 0.97 & 0.98 \\
\hline
\end{tabular}

The element in the first row in each box represents the results based on Simulations (10,000 replications) in case of ratio of the maximum likelihood functions denoted by RMLS and the number in bracket immediately below represents the result obtained by 
using asymptotic results in case of ratio of the maximum likelihood functions denoted by RMLAC. The element in third row represent the RMKLD.

Table 10: The Probability of Correct Selection Based on Simulations and also Based on Asymptotic Results When the Null Distribution is log-logistic distribution

\begin{tabular}{|c|c|c|c|c|c|c|}
\hline \multirow[b]{2}{*}{$\sigma$} & \multicolumn{6}{|c|}{$\mathrm{n}$} \\
\hline & PCS & 20 & 40 & 60 & 80 & 100 \\
\hline \multirow[t]{3}{*}{2.2} & RMLS & 0.51 & 0.56 & 0.64 & 0.67 & 0.70 \\
\hline & RMLAR & $(0.47)$ & $(0.53)$ & $(0.57)$ & $(0.62)$ & $(0.66)$ \\
\hline & RMKLD & 0.57 & 0.62 & 0.68 & 0.73 & 0.75 \\
\hline \multirow[t]{3}{*}{2.5} & RMLS & 0.53 & 0.58 & 0.65 & 0.69 & 0.72 \\
\hline & RMLAR & $(0.48)$ & $(0.53)$ & $(0.58)$ & $(0.63)$ & $(0.68)$ \\
\hline & RMKLD & 0.58 & 0.64 & 0.68 & 0.74 & 0.77 \\
\hline \multirow[t]{3}{*}{3.0} & RMLS & 0.54 & 0.59 & 0.66 & 0.70 & 0.74 \\
\hline & RMLAR & $(0.51)$ & $(0.55)$ & $(0.58)$ & $(0.64)$ & $(0.69)$ \\
\hline & RMKLD & 0.61 & 0.64 & 0.70 & 0.73 & 0.78 \\
\hline \multirow[t]{3}{*}{3.2} & RMLS & 0.55 & 0.61 & 0.67 & 0.73 & 0.75 \\
\hline & RMLAR & $(0.54)$ & $(0.57)$ & $(0.61)$ & $(0.69)$ & $(0.73)$ \\
\hline & RMKLD & 0.62 & 0.68 & 0.72 & 0.77 & 0.80 \\
\hline \multirow[t]{3}{*}{3.5} & RMLS & 0.57 & 0.64 & 0.70 & 0.76 & 0.77 \\
\hline & RMLAR & $(0.57)$ & $(0.62)$ & $(0.66)$ & $(0.69)$ & $(0.75)$ \\
\hline & RMKLD & 0.65 & 0.70 & 0.74 & 0.79 & 0.82 \\
\hline \multirow[t]{3}{*}{4.00} & RMLS & 0.59 & 0.66 & 0.73 & 0.78 & 0.81 \\
\hline & RMLAR & $(0.61)$ & $(0.63)$ & $(0.67)$ & $(0.71)$ & $(0.78)$ \\
\hline & RMKLD & 0.69 & 0.71 & 0.77 & 0.81 & 0.83 \\
\hline
\end{tabular}


The element in the first row in each box represents the results based on Simulations (10,000 replications) in case of ratio of the maximum likelihood functions denoted by RMLS and the number in bracket immediately below represents the result obtained by using asymptotic results in case of ratio of the maximum likelihood functions denoted by RMLAC. The element in third row represent the RMKLD.

It is quite clear from Tables 9 and 10 that as the sample size increases the PCS increases as expected. It is also clear that as the shape parameter moves away from 1, the PCS increases. Even when the sample size is 20.

Interestingly, when the null distribution is gamma distribution, then the PCS based on MC simulation, AR and RMKLD is found to be significantly higher than the other case particularly for small sample sizes. For example, when the sample size is 20, and null distribution is gamma, the PCS for MC, AR and RMKLD are 0.77, 0.75 and 0.83 respectively. But when the null distribution is log-logistic, for the same sample size the PCS for MC, AR and RMKLD are $0.51,0.47$ and 0.57 respectively.

The asymptotic results work reasonable well for both the distributions and for all possible ranges of the parameters. From the simulation study it is recommended that the asymptotic results can be used quite effectively even when the sample size is as small as 20 for all possible choices of the shape parameters. Moreover, as shape parameter increases, the PCS increases.

Also from Tables 9 and 10 notice that, the RMKLD works better than RML, because it generates higher PCS (about 3-9 \%) in particular for small sample size. In other words, the error type I for RMKLD is remarkably less, compared to the RML one. For instance, consider in table 9 the case at $\alpha=1.2$. The PCS for RMKLD is $83 \%$ and $77 \%$ for RML which indicates the error type I equals to $17 \%$ for RMKLD and $23 \%$ for RML. also consider in table 10 the case $\sigma=2.2$ The PCS for RMKLD is $57 \%$ and $51 \%$ for RML which indicates the error type I equals to $43 \%$ for RMKLD and $49 \%$ for RML It also found that both methods behave similarly, for example, as sample size increases the PCS capture higher values, as expected.

\subsection{Data Analysis}

For illustrative purposes two data sets will be analyzed using RML method and some goodness of fit tests.

Data Set1: ( Lawless, 1982, p. 228) it represents the number of revolution before failure of each of 23 ball bearings in the life tests and they are as follows: 17.88, 28.92, 33.00, 41.52 , 42.12, 45.60, 48.80, 51.84, 51.96, 54.12, 55.56, 67.80, 68.44, 68.64,68.88, 84.12, 93.12, 98.64, 105.12, 105.84, 127.92, 128.04, 173.40.

In this case the two fitted distributions have the following MLEs: log-likelihood values are as follows. Gamma: $\hat{\lambda}=3.7138, \hat{\alpha}=19.449, \operatorname{LL}_{\mathrm{GA}}(\hat{\lambda}, \hat{\alpha})=-113.0274$, LogLogistic: $\hat{\sigma}=3.0591, \hat{\varepsilon}=60.639, \quad \operatorname{LL}_{\mathrm{LL}}(\hat{\varepsilon}, \hat{\sigma})=-113.3662$. 
Therefore by using the method of the Maximized Likelihood Procedure

$$
T=\ln L_{G A}-\ln L_{L L}=0.3388>0
$$

Then gamma distribution is the preferred one. The closeness of results may be caused because the sample size is not very large.

Moreover by computing the goodness of fit tests at $\hat{\lambda}=3.7138, \hat{\alpha}=19.449$ and $\hat{\sigma}=3.0591, \hat{\varepsilon}=60.639$. Therefore, it is nearly shows that the fitted gamma is much closer to the empirical distribution function than the log-logistic distribution. Results are reported in Table 11 .

Table 11: Goodness of Fit for Lawless Data Set

\begin{tabular}{|c|c|c|c|c|}
\hline$\#$ & Distribution & Kolmogorov Smirnov & Anderson Darling & Chi-Squared \\
\hline 1 & Log-Logistic & 0.10847 & 0.30294 & 0.93098 \\
\hline 2 & Gamma & 0.11878 & 0.20569 & 0.67705 \\
\hline
\end{tabular}

Data Set 2: (Linhart and Zucchini, 1986, p. 69) represent the failure times of 30 air conditions of an airplane (in hours): 23, 261, 87, 7, 120, 14, 62, 47, 225,71, 246, 21, 42, $20,5,12,120,11,3,14,71,11,14,11,16,90,1,16,52,95$.

In this case the two fitted distributions have the following MLEs: log-likelihood values are as follows. Gamma: $\hat{\lambda}=0.68741, \hat{\alpha}=86.702, \mathrm{LL}_{\mathrm{GA}}(\hat{\lambda}, \hat{\alpha})=-151.706$.

Log-Logistic: $\hat{\sigma}=1.2024, \hat{\varepsilon}=26.629, \operatorname{LL}_{\mathrm{LL}}(\hat{\varepsilon}, \hat{\sigma})=-152.3468$.

Therefore by using the method of the Maximized Likelihood Procedure

$$
T=\ln L_{G A}-\ln L_{L L}=0.9408>0
$$

Then gamma distribution is the preferred one. Moreover by computing the goodness of fit tests at $\hat{\lambda}=0.68741, \hat{\alpha}=86.702$ and $\hat{\sigma}=1.2024, \hat{\varepsilon}=26.629$. Therefore, it is nearly shows that the fitted logistic is much closer to the empirical distribution function than the gamma distribution. Results are reported in Table 12.

Table 12: $\quad$ Goodness of Fit for Linhart and Zucchini Data Set

\begin{tabular}{|c|l|c|c|c|}
\hline$\#$ & Distribution & Kolmogorov Smirnov & Anderson Darling & Chi-Squared \\
\hline 1 & Log-Logistic & 0.1234 & 0.49039 & 2.6152 \\
\hline 2 & Gamma & 0.13432 & 0.57009 & 1.7764 \\
\hline
\end{tabular}




\section{Conclusion}

The problem of discriminating gamma and log-logistic is considered in case of progressive type II censoring. The RML test is used to discriminate between them. Some simulation experiments were performed to see how the PCS under each model work for small sample sizes. It can be observed that the PCS works quite well even for small sample sizes. Interestingly, when Gamma is the true distribution, then the PCS based on simulation is found to be significantly higher than the other case particularly for small sample sizes. Also using real data life and applying RML test we found that gamma model is chosen for this data set.

As a special case of progressive type II censoring when censoring scheme $R_{i}=0$, The problem of discriminating between gamma and log-logistic in case of complete samples is considered. The RML and the RMKLD are used to discriminate between them. The asymptotic results are used to estimate the PCS which is used calculate the minimum sample size required for discriminating between two distributions. The PCS using simulations with the asymptotic results and RMKLD is compared and it is observed that even when the sample size is very small the asymptotic results work quite well for a wide range of the parameter space. Also it noticed that when the null distribution is gamma distribution, then the PCS is found to be significantly higher than the other case particularly for small sample sizes. Two Real data life are analyzed and applying RML test we found that gamma model is chosen for these two data sets.

\section{References}

1. Ashkar, F. and Aucoin, F., (2012). Choice between competitive pairs of frequency models for use in hydrology: a review and some new results. Hydrological Sciences Journal, vol. 57, no.6, 1092-1106.

2. Atkinson, A. (1970). A method for discriminating between models. Journal of the Royal Statistical Society, series B, no.32, 323-353.

3. Bain, L. J., and Engelhardt, M., (1980). Probability of correct selection of Weibull versus gamma based on likelihood ratio. Communications in Statistics, Theory and Methods, vol. 9, no.6, 375-381.

4. Balasooriya, U. and Balakrishnan, N. (2000). "Reliability sampling plan for lognormal distribution", IEEE Transactions on Reliability, vol. 49, 199 - 203.

5. Balakrishnan, N. and Aggarwala, R. (2000). Progressive censoring: theory, methods and applications. Birkhauser, Boston.

6. Balakrishnan, N. and Kannan, N. (2001). "Point and interval estimation for parameters of the logistic distribution based on progressively type-II censored data", Handbook of Statistics, vol. 20, North Holland, Amsterdam.

7. Balakrishnan, N., Kannan, N., Lin, C.T. and Ng, H. (2003). Point and interval estimation for Gaussian distribution based on progressively Type-II censored samples. IEEE Trans. Reliab., 52, 90-95. 
8. Balakrishnan, N., Kannan, N., Lin, C.T. and Wu, S.J.S. (2004). "Inference for the extreme value distribution under progressive type-II censoring", Journal of Statistical Computations and Simulations, 25- 45.

9. Balakrishnan, N. and Sandhu, R.A. (1995). “A simple algorithm for generating progressively type-II generated samples”, American Statistician, vol. 49, 229 230.

10. Balakrishnan, N. (2007). "Progressive Censoring Methodology: An Appraisal", (with discussions), TEST, vol. 16, no. 2, $211-296$.

11. Bedar. AL-E. R. (2009). On the distribution of the ratio of the maximized likelihoods (RML) statistic. M.Sc in statistic. Institute of Statistical Studies and Research. Cairo University.

12. Block, A.D., Leemis, L.M., (2008). Parametric model discrimination for heavily censored survival data. IEEE Transactions on Reliability, vol.57, no.2, 248-259.

13. Bromideh, A. A. (2012). Discriminating Between Weibull and Log-Normal Distributions Based on Kullback-Leibler Divergence, Istanbul University Econometrics and Statistics e-Journal, Vol.16, no.1, 44-54.

14. Bromideh, A. A., Valizadeh, R. (2013). Discrimination between Gamma and LogNormal Distributions by Ratio of Minimized Kullback-Leibler Divergence. Pak.j.stat.oper.res. Vol. IX, no.4, 441-451.

15. Cain, S.R., (2002). Distinguishing between lognormal and Weibull distributions. IEEE Transactions on Reliability, vol.51, no.1, 32-38.

16. Cohen, A.C. (1965). Maximum likelihood estimation in the Weibull distribution based on complete and censored samples. Technometrics, 7, 579-588.

17. Cox, D. R. (1961). Tests of separate families of hypotheses. Proceeding of the Fourth Berkely Symposium in Mathematical Statistics and Probability, Berkely, University of California Press, 105-123.

18. Cox, D. R. (1962). Further results on tests of separate families of hypo-theses. Journal of the Royal Statistical Society, Series B, vol. 24, 406-424.

19. Dey, A. K., and Kundu, D. K. (2009). Discriminating among the log-normal, Weibull and generalized exponential distributions. IEEE Transactions on Reliability, vol. 58, no. 3, 416-424.

20. Dey, A. K., and Kundu, D. K. (2010). Discriminating between the log-normal and log-logistic distributions. Communications in Statistics, Theory and Methods, vol. $39,280-292$.

21. Dey, A. K., and Kundu, D. K. (2012). Discriminating between the Weibull and Log-normal distributions for type-II censored data. Statistics, vol. 46, no. 2, 197 214.

22. Dumonceaux, R., and Antle., C.E. (1973). Discriminating between the log-normal and Weibull distribution. Technometrics, vol. 15, no.4, 923-926. 
23. Dumonceaux, R., Antle, C., and Hass, G. (1973). Liklihood ratio test for discrimination between two models with unknown location and scale parameters. Technometrics, 19-27.

24. Elsherpieny, A. E., Ibrahim, N. S. And Radwan U. N. (2013). Discriminating Between Weibull and Log-logistic Distributions. International Journal of Innovative Research in Science, Engineering and Technology. Vol. 2, no.8, 33583371.

25. Fearn, D. H., and Nebenzahl, E. (1991). On the maximum likelihood ratio method of deciding between the Weibull and gamma distributions. Communications in Statistics, Theory and Methods, vol. 20, 579-593.

26. Firth, D. (1988). Multiplicative errors: log-normal or gamma? Journal of the Royal Statistical Society, Series B. 2, 266-268.

27. Gupta, R. D., Kundu, D. K. and Manglick, A. (2001). Probability of correct selection of gamma versus generalized exponential or generalized exponential versus Weibull. Proceeding of statistics canada: Recent advanced in statistical methods, Edited by Y.P chaubey, Imperial college press, 147-156

28. Gupta, R. D., and Kundu, D. K. (2003). Discriminating between Weibull and generalized exponential distributions. Journal of Computational Statistics and Data Analysis, vol.43, 179 - 196.

29. Gupta, R. D., and Kundu, D. k. (2004). Discriminating between the gamma and generalized exponential distributions. Journal of Statistical Computation and Simulation, vol. 74, no.2, 107-121.

30. Kappenman, R. F. (1982). On a method for selecting a distributional model. Communication in Statistics, Theory and Methods, vol.11, 663-672.

31. Kim, D.H., Lee, W.D., Kang, S.G., (2000). Bayesian model selection for life time data under type II censoring. Communications in Statistics-Theory and Methods, vol.29, no.12, 2865-2878.

32. Kim, J.S. and Yum, B-J., (2008). Selection between Weibull and log-normal distributions: A comparative simulation study. Computational Statistics and Data Analysis, vol. 53, 477 - 485.

33. [33] Kundu, D. K., and Manglick, A. (2005). Discriminating between the lognormal and gamma distributions. Journal of the Applied Statistical Sciences, vol.14, 175-187.

34. Kundu, D., Raqab, M. Z. (2007). Discriminating Between the Generalized Rayleigh and Log-Normal Distribution. Statistics, vol.41 no.6, 505-515.

35. Lawless, J. F. (1982). Statistical Models and Methods for Lifetime Data. John Wiley and Sons, New York.

36. Linhart, H., Zucchini, W. (1986). Model selection. New York: Wiley.

37. Mann, N.R. (1971), "Best linear invariant estimation for Weibull parameters under progressive censoring”, Technometrics, vol. 13, 521 - 533. 
38. Mousa, M. and Jaheen, Z. (2002). Statistical inference for the Burr model based on progressively censored data. An international Computers \& Mathematics with applications, 43, 1441-1449.

39. Nelson, W.B. (1982). Applied life data analysis, John Wiley. New York.

40. Pandey, M., Ferdous, J. and Uddin, M. B. (1991). Selection of probability distributions for life testing data. Communications in Statistics, Theory and Methods, 20(4), 1373-1388.

41. Pascual, F. G. (2005). Maximum likelihood estimation under misspecified lognormal and Weibull distributions. Communications in Statistics, Simulation and Computations, vol. 34, 503-524.

42. Pereira, B. de B. (1977). A note on the consistency and on the finite sample comparisons of some tests of separate families of hypotheses. Biometrika, vol. 64, 109-113.

43. Rao, S. B. and Kantam L. R. R. (2014). Discriminating between log-logistic and rayleigh distributions, Pakistan Journal of Statistics and Operation Research, Vol.X, no.1,1-7.

44. Raqab, M. Z. (2013). Discriminating between the generalized Rayleigh and Weibull distributions. Journal of Applied Statistics, vol. 40, no.7, 1480-1493.

45. Siswadi, Quesenberry, C.P. (1982). Selecting among Weibull, lognormal and gamma distributions using complete and censored samples. Naval Research Logistics Quarterly 29. no.4, 557-569.

46. Thomas, D.R. and Wilson, W.M. (1972), "Linear order statistic estimation for the two parameter Weibull and extreme value distributions from type-II progressively censored samples", Technometrics, vol. 14, 679 - 691.

47. Viveros, R. and Balakrishnan, N. (1994). Interval estimation of parameters of life from progressively censored data, Technometrics 36, 84-91.

48. White, H. (1982). Regularity conditions for Cox's test of non-nested hypotheses. Journal of Econometrics, vol.19, 301-318.

49. Wiens, B. L. (1999). When log-normal and gamma models give different results: a case study. American Statistician, vol. 53, 89-93. 